\title{
Tecnologia educacional em saúde para pacientes em tratamento quimioterápico ambulatorial
}

\author{
Health educational technology for patients in \\ ambulatorial chemotherapy treatment
}

\author{
Daniele Lima dos Anjos Reis ${ }^{1,2}$, Silvia Maria Azevedo dos Santos³, Kátia Simone Kietzer 2,4 \\ ' Universidade do Estado do Pará, Tucuruí (PA), Brasil. \\ ${ }^{2}$ Programa de Pós-graduação Ensino em Saúde da Amazônia, Centro de Ciências Biológicas e da Saúde \\ Universidade do Estado do Pará, Belém (PA), Brasil. \\ Universidade Federal de Santa Catarina, Florianópolis (SC), Brasil. \\ ${ }^{4}$ Programa de Pós-graduação em Cirurgia e Pesquisa Experimental, Centro de Ciências Biológicas e da Saúde, \\ Universidade do Estado do Pará, Belém (PA), Brasil.
}

\section{RESUMO}

Introdução: A quimioterapia antineoplásica é uma modalidade terapêutica sistêmica para tratamento do câncer, um grave problema de saúde pública. Nesse contexto, a educação em saúde representa um importante instrumento facilitador para a capacitação da comunidade, destacando-se o uso de tecnologias educativas durante as consultas de enfermagem em oncologia. Assim, objetivou-se elaborar material educativo na forma de cartilha com orientações destinadas a pacientes que realizam tratamento quimioterápico ambulatorial na Unidade de Atendimento de Alta Complexidade em Oncologia (Unacon) Dr. Vitor Moutinho, em Tucuruí-PA. Métodos: O produto enquadrou-se na categoria de Tecnologia de Educação, sendo destinado tanto aos pacientes adultos com diagnóstico de câncer que realizam tratamento quimioterápico ambulatorial quanto aos profissionais enfermeiros que estão envolvidos no atendimento a esses pacientes. Sua construção ocorreu entre os meses de novembro de 2013 e maio de 2014, sendo atualizado em 2017. Para isso, realizou-se uma revisão da literatura científica sobre o tratamento quimioterápico antineoplásico e materiais educativos em saúde, sendo selecionados 03 livros didáticos, 05 manuais/protocolos de outras instituições e 01 monografia. Resultados e Discussão: Foi adotada uma linguagem simples e coloquial e a estratégia de interação com o leitor, visando aproximar ao máximo o público-alvo com os assuntos abordados. A cartilha elaborada contém as principais dúvidas apresentadas por pacientes oncológicos submetidos ao tratamento quimioterápico e os conteúdos de interesse extraídos foram organizados nas seguintes categorias: 1- Conhecimentos sobre o câncer; 2- Conhecimentos sobre as formas de tratamento e 3- Conhecimentos sobre a quimioterapia. Conclusões: Percebeu-se a relevância dos materiais educativos, pois eles favorecem o processo interativo entre enfermeiro, paciente ou familiar, auxiliam no diálogo e proporcionam a valorização da humanização da assistência. Ainda, por meio do produto gerado, será possível sugerir ações para contribuir com o processo de estruturação dos serviços de oncologia em Tucuruí-PA.

PALAVRAS-ChAVE: Câncer. Quimioterapia. Tecnologia educacional. Educação em saúde.

\section{CORRESPONDÊNCIA}

Daniele Lima dos Anjos Reis

Universidade do Estado do Pará

Rua Tocantins, 55, Vila Tropical,

CEP 68455-761, Tucuruí (PA), Brasil

anjo.daniele@hotmail.com

\section{FONTE DE FINANCIAMENTO}

Nenhuma.

\section{CONFLITO DE INTERESSE}

Os autores declararam não

haver conflitos de interesse.

O estudo foi realizado na Universidade do Estado Pará, núcleo de Tucuruí e na Unidade de Atendimento de Alta Dr. Vitor Moutinho, Tucuruí (PA), Brasil.

\section{ABSTRACT}

Introduction: Antineoplastic chemotherapy is a systemic therapeutic modality for the treatment of cancer, a serious public health problem. In this context, health education represents an important facilitating tool for community empowerment, with emphasis on the use of educational technologies during nursing consultations in oncology. Thus, the objective was to develop educational material in the form of a booklet with guidelines intended for patients who undergo chemotherapy treatment at the High Complexity Oncology Care Unit (Unacon) Dr. Vitor Moutinho in Tucuruí-PA. Methods: The product was included in the category of Education Technology, and it is intended for both adult patients with cancer diagnosis who undergo chemotherapy treatment in the outpatient clinic and the nursing professionals who are involved in the care of these patients. Its construction took place between the months of November 2013 and May 2014 and was updated in 2017. For this, a review of the scientific literature on the chemotherapy treatment of antineoplastic and educational materials in health was carried out, being selected 03 textbooks, 05 manuals / Protocols of other institutions and 01 monograph. Results and Discussion: A simple and colloquial language and the strategy of interaction with the reader were adopted, aiming 
at bringing the target public as close to the subjects as possible. The elaborated primer contains the main doubts presented by oncology patients submitted to the chemotherapy treatment and the contents of interest extracted were organized in the following categories: 1- Knowledge about cancer; 2-Knowledge about the forms of treatment and 3-Knowledge about the chemotherapy. Conclusions: The relevance of the educational materials was perceived, since they favor the interactive process between nurse, patient or family, help in the dialogue and provide the valorization of the humanization of care. Also, through the generated product, it will be possible to suggest actions to contribute to the structuring process of the oncology services in Tucuruí-PA.

KEYWORDS: Cancer. Chemotherapy. Educational technology. Health education.

\section{Introdução}

Considerado um grave problema de saúde pública, câncer é o nome dado a um conjunto de mais de 100 tipos diferentes de doenças que têm em comum o crescimento desordenado de células anormais com potencial invasivo. Sua origem se dá por condições multifatoriais, que podem agir em conjunto ou em sequência para iniciar ou promover a carcinogênese ${ }^{1}$.

As opções de tratamento oferecidas aos pacientes oncológicos deverão ser baseadas em metas realistas e tangíveis de acordo com cada tipo de câncer. Como terapêutica são utilizadas a cirurgia e a radioterapia, como modalidade de tratamento localizado, e a quimioterapia antineoplásica e terapias que utilizam moduladores genéticos, como métodos de tratamento sistêmicos ${ }^{2}$.

O conceito de quimioterapia para as mais variadas neoplasias tem sua origem há pelo menos 1.500 anos, remontando às civilizações egípcia e grega. A partir do final do século XIX, com a descoberta e sintetização de novas drogas e sua associação houve o vislumbre de encarar o câncer como uma doença potencialmente curável. Em contraposição a esta evolução na pesquisa, os pacientes ficaram sujeitos a uma exposição a doses maciças de medicações citotóxicas que podem desencadear efeitos adversos dos mais variados, inclusive com risco de morte. Isto levou à reflexão de levar em consideração os riscos e benefícios ${ }^{3}$.

Diante do impacto do diagnóstico, os pacientes e familiares chegam abalados para a consulta de enfermagem em oncologia. Neste momento, percebe-se que há maior dificuldade em assimilar de forma eficiente as informações transmitidas verbalmente. Considerando a complexidade que envolve o uso de quimioterápicos, é imprescindível que os pacientes submetidos a esta modalidade terapêutica estejam totalmente informados acerca das questões inerentes ao tratamento.

Nesta perspectiva, a educação em saúde representa um importante instrumento facilitador para a capacitação da comunidade, contribuindo para a promoção da saúde, mudança de comportamento e estimulação do autocuidado. As abordagens mais modernas têm destacado o uso da tecnologia educativa na saúde, onde a tecnologia se manifesta como objetos e recursos antigos e atuais (como os materiais educativos/informativos) que têm a finalidade de aumentar e melhorar o tratamento e o cuidado por meio da prática em saúde, manifestando-se também na forma de conhecimentos e habilidades em saúde associadas com o uso e a aplicação dos recursos e objetos usados pelos profissionais ${ }^{4}$.

Para o desenvolvimento desse tipo de material educativo

[...] é necessária uma abordagem com uma linguagem acessível para facilitar o entendimento e cooperação no tratamento, incentivando o paciente a enfrentar as mudanças advindas com a doença e a alcançar o bem-estar $[\ldots]^{4}$ (p. 14).

Assim, apesar de algumas restrições e limitações para o uso de material impresso devido à baixa escolaridade de determinada parcela populacional, esse recurso é amplamente utilizado para se veicular mensagens de saúde e para se facilitar o processo ensino-aprendizagem no contexto oncológico. Essa estratégia é capaz de estimular o paciente e seu familiar a deixarem o papel de sujeitos passivos e passarem a interagir com a equipe expondo dúvidas, angústias e sentimentos durante o tratamento. Neste contexto, o enfermeiro é considerado elemento-chave na difusão 
do conhecimento, sendo que este profissional deve estar preparado e capacitado para atender às necessidades da clientela oncológica e tornar mais fácil o enfrentamento deste sério problema de saúde pública.

Diante do exposto, é possível inferir que o processo educativo proposto por este trabalho poderá contribuir positivamente para o enriquecimento das consultas de enfermagem realizadas pelos enfermeiros que atuam na Unidade de Atendimento de Alta Complexidade em Oncologia (Unacon) Dr. Vitor Moutinho do município de Tucuruí-PA, pois deverá incentivar os profissionais a trabalharem com a prática da aprendizagem colaborativa, crítica e reflexiva dentro de um contexto educativo. Além disso, contribuirá para a obtenção de resultados favoráveis no tratamento de pacientes atendidos nesta unidade oncológica, pois acredita-se que um paciente bem informado, que compreende o que está acontecendo com sua saúde e o porquê de todas as ações e procedimentos realizados, tende a aderir mais facilmente à terapêutica instituída, resultando em melhoria de sua qualidade de vida e em maiores possibilidades de cura.

Desta forma, o objetivo geral deste trabalho foi elaborar um material educativo na forma de cartilha com orientações destinadas a pacientes que realizam tratamento quimioterápico ambulatorial na Unacon do município de Tucuruí-PA.

\section{Métodos}

O produto gerado por este estudo constituiu-se em um material educativo, portanto, a tecnologia que melhor define o resultado é a Tecnologia de Educação. As tecnologias de educação apontam formas de colaborar com a formação de uma consciência para a vida saudável ${ }^{5}$.

O material educativo é destinado à Unacon do município de Tucuruí-PA, para ser utilizado tanto pelos pacientes adultos com diagnóstico de câncer que realizam tratamento quimioterápico ambulatorial quanto pelos profissionais enfermeiros que estão envolvidos no atendimento a esses pacientes. Entende-se por Unacon o hospital dotado de instalações físicas, condições técnicas, equipamentos e recursos humanos adequados à prestação de assistência especializada de alta complexidade para o diagnóstico e tratamento das neoplasias mais prevalentes no país, como os cânceres de colo de útero, mama, próstata, estômago, cólon e reto ${ }^{6}$.

A construção inicial deste trabalho se deu entre os meses de novembro de 2013 e maio de 2014, sendo atualizado nos meses de junho e agosto de 2017, para posterior aprovação, validação e utilização do mesmo. Realizou-se uma revisão da literatura científica sobre o tratamento quimioterápico antineoplásico e materiais educativos em saúde. A principal vantagem da pesquisa bibliográfica reside no fato de que ela permite investigar uma ampla gama de fenômenos por meio da busca em materiais já elaborados, possibilitando o aprimoramento de ideias e conceitos ${ }^{7}$.

A fim de identificar os textos que abordem o tema em questão, foram coletados dados por meio de instrumentos como livros didáticos; manuais/protocolos de outras instituições, monografias; sites especializados, como o do Instituto Nacional de Câncer (INCA) e de outros hospitais que lidam com o tratamento do câncer; e artigos científicos disponíveis na Biblioteca Virtual em Saúde (BVS), nas seguintes bases: Scientific Electronic Library Online (Scielo) e Literatura Latino-Americana e do Caribe em Ciências da Saúde (Lilacs). Para a busca das obras foram utilizados os seguintes descritores: câncer, quimioterapia, tecnologia educacional, cartilha educativa, orientações para quimioterapia.

Adotou-se como critérios de inclusão: obras nacionais, em meio impresso ou eletrônico sobre a temática, publicadas no período de 2008 a 2016. Como critérios de exclusão adotou-se: obras com conteúdos repetidos. 
Durante a pesquisa para a elaboração da cartilha foram selecionados 03 livros didáticos, 05 manuais/protocolos de outras instituições e 01 monografia.

Posteriormente, procedeu-se à leitura cuidadosa com análise do material selecionado, extraindo os conteúdos de interesse e os agrupando por eixos temáticos, conforme o que se pretendia abordar na construção do material educativo. Em um segundo momento buscou-se adequar a linguagem científica para a mais próxima do entendimento da população leiga, objetivando apresentar de forma clara e objetiva as informações. Em um terceiro momento procedeu-se à análise crítica e reflexiva acerca da forma com que os conteúdos preparados seriam organizados e a lógica de sua apresentação. A cartilha foi confeccionada utilizando-se o programa Microsoft Power Point 2010.

\section{Resultados e discussão}

Para facilitar a criação da cartilha educativa, os conteúdos de interesse foram extraídos e organizados nas seguintes categorias: 1- Conhecimentos sobre o câncer; 2-Conhecimentos sobre as formas de tratamento; 3- Conhecimentos sobre a quimioterapia.

Optou-se por iniciar a cartilha com uma apresentação do material, a fim de que os pacientes se familiarizem com as informações que irão receber ao realizarem a leitura e conheçam o objetivo do seu fornecimento. Além disso, a presença do sumário facilita a observação e busca dos temas tratados na cartilha.

É válido enfatizar que foi adotada uma linguagem simples e coloquial para a elaboração do material educativo, pois acredita-se que a "[...] produção de um material didático precisa ser cuidadosa em relação à linguagem de seus textos, qualquer que seja o nível do curso ou grau de escolaridade de seu público-alvo"4 (p. 41).

Nesse contexto, o enfermeiro tem a responsabilidade de prestar informações claras aos pacientes oncológicos ou aos seus responsáveis, sempre considerando os diferentes níveis de escolaridade e conhecimento de cada um, oferecendo alternativas terapêuticas e de cuidado, mostrando os riscos e benefícios inerentes a cada uma delas e certificando-se de que houve a compreensão dos conteúdos prestados, o que pode ser facilitado pelo uso de material informativo ${ }^{8}$.

Utilizou-se, ainda, a estratégia de interação com o leitor, pois, é necessário aproximar ao máximo o público-alvo com os assuntos abordados, sempre na intenção de atraí-los para um diálogo ${ }^{8}$, utilizando-se de expressões como "você sabe como se cuidar?", "atenção", "lembre-se", "importante saber", etc., de maneira que estas além de chamar a atenção do leitor, proporciona maior adesão à tecnologia educativa.

\section{Conhecimentos sobre o câncer}

Esta categoria corresponde à página 04 da cartilha e reúne informações práticas sobre a definição do câncer, tipos de câncer e principais fatores de risco.

A importância de o paciente saber mais sobre sua patologia, qualquer que seja ela, está no fato de que o conhecimento é capaz de desmitificar crenças, tabus e ideias preconcebidas, favorecendo o sucesso do tratamento. Complementando esta temática, Sousa $^{8}$ (p. 57) em seu trabalho sobre a Insuficiência Renal Crônica (IRC) diz que

[...] o conhecimento sobre a IRC é importante para o desenvolvimento do autocuidado, pois ajuda no tratamento e com isso previne invalidez ou morte. O conhecimento sobre a doença renal crônica (DRC), as necessidades de bem-estar e o tratamento para esse agravo possibilita ao cliente: entendimento e aceitação, favorecendo comportamentos de autocuidado; maior adesão às intervenções terapêuticas, inclusive de enfermagem; diminuição das intercorrências durante o procedimento dialítico e, consequentemente, promoção de sua qualidade de vida, mesmo convivendo com a DRC. 
Assim, constata-se que a falta de conhecimento por parte dos pacientes pode gerar dificuldade na compreensão do tratamento e seus efeitos colaterais, bem como no enfretamento da patologia, culminando muitas vezes no abandono do mesmo.

\section{Conhecimentos sobre as formas de tratamento}

Esta categoria corresponde à página 05 do material, e nela foram selecionadas informações simples sobre a terapêutica do câncer, que é composta basicamente por três modalidades: a cirurgia (sendo o tipo de tratamento mais antigo, objetivando eliminar o tumor e levar à cura, podendo ser associada à radioterapia e quimioterapia), a radioterapia (na qual o tratamento é por meio da radiação, aplicada diretamente na área afetada), e a quimioterapia (tratamento que utiliza agentes químicos com a finalidade de destruir as células cancerígenas ou inibir seu crescimento e proliferação) $)^{9}$.

O enfermeiro, por estar em constante contato com o paciente, exerce um importante papel de educador junto a ele, fornecendo informações relacionadas à terapêutica proposta ${ }^{10}$. Destarte, é essencial que o paciente tome conhecimento de todas as possíveis formas de tratamento do câncer e saiba em qual(s) modalidade(s) será submetido, pois o tratamento indicado para cada paciente vai depender das características específicas do tumor que apresenta, sua localização, tamanho, presença ou não de metástase ${ }^{9}$.

\section{Conhecimentos sobre a quimioterapia}

Foram reunidos nesta categoria, correspondente às páginas 06 a 21, conteúdos relativos à quimioterapia antineoplásica, como sua definição, tempo de tratamento, formas de administração, efeitos colaterais, sinais e sintomas de alerta e outras orientações relevantes ao sucesso do tratamento.

"O paciente e seu cuidador devem ser informados sobre todos os aspectos da terapia biológica ou antineoplásica, incluindo efeitos físicos e psicológicos, reações adversas, riscos e benefícios" ${ }^{\prime \prime}$ (p. 254). Além da necessidade de serem informados sobre esses aspectos, os pacientes em tratamento quimioterápico devem ser estimulados ao autocuidado, pois, a quimioterapia é uma forma de tratamento que causa diversas toxicidades no organismo humano, carecendo de intervenção não apenas medicamentosa, mas também de cuidados que podem ser tomados pelo próprio paciente ${ }^{11}$.

O estudo realizado por Barbosa e Telles ${ }^{10}$ mostrou um déficit de conhecimento no que se refere à quimioterapia e que as principais dúvidas dos pacientes entrevistados eram sobre os efeitos colaterais da terapêutica, reforçando o fato de eles interferirem na vida cotidiana. Além disso, deve-se levar em consideração que, com a tendência atual da desospitalização, o tratamento quimioterápico é realizado nos serviços ambulatoriais, e os efeitos colaterais acabam surgindo em casa, cabendo ao próprio paciente, familiar ou cuidador realizarem os cuidados necessários ${ }^{8}$. Desta maneira, sendo os efeitos colaterais uma das maiores causas de abandono do tratamento, faz-se necessário propiciar informações direcionadas primordialmente ao seu controle.

Assim, levando em consideração o exposto e as informações contidas no material educativo elaborado, o paciente em quimioterapia conhecerá os principais aspectos relativos ao seu tratamento, com ênfase nos efeitos colaterais das medicações e, principalmente, saberá o que fazer diante desses efeitos e quando procurar o serviço de urgência, sendo estas umas das prioridades no tratamento oncológico: incluir o paciente em seu plano terapêutico.

A Tabela 1 mostra, de forma simplificada, a estrutura física da cartilha de orientações ao paciente em quimioterapia. 
Tabela 1. Estrutura da cartilha de orientações ao paciente em quimioterapia.

\begin{tabular}{|c|c|c|}
\hline TEMA & CONTEÚDO & $\begin{array}{l}\mathbf{N}^{\circ} \text { DE } \\
\text { PÁGINAS }\end{array}$ \\
\hline Sumário & Temas tratados na cartilha e numeração das páginas & 01 \\
\hline Apresentação & Objetivos da cartilha & 01 \\
\hline O que é o câncer? & $\begin{array}{l}\text { Definição do câncer, principais tipos de câncer no Pará } \\
\text { e causas/fatores de risco }\end{array}$ & 01 \\
\hline Quais as formas de tratamento? & $\begin{array}{l}\text { Definição das modalidades terapêuticas atuais para o } \\
\text { câncer }\end{array}$ & 01 \\
\hline O que é quimioterapia? & Definição do tratamento medicamentoso & 01 \\
\hline $\begin{array}{l}\text { Como é aplicada a } \\
\text { quimioterapia? }\end{array}$ & Forma de administração dos quimioterápicos & 01 \\
\hline $\begin{array}{l}\text { Quais reações posso ter durante } \\
\text { o tratamento? }\end{array}$ & $\begin{array}{l}\text { Principais reações e condutas: náuseas e vômito; } \\
\text { diarreia; intestino preso; sensação de estômago muito } \\
\text { cheio; alterações na pele e unhas; queda de cabelos e } \\
\text { pêlos do corpo; alterações na boca; fraqueza, cansaço e } \\
\text { anemia; problemas com a coagulação; infecção }\end{array}$ & 09 \\
\hline $\begin{array}{l}\text { Quando devo procurar um } \\
\text { hospital com urgência? }\end{array}$ & Sinais e sintomas de alerta & 01 \\
\hline Dicas e orientações importantes & $\begin{array}{l}\text { Informações relevantes sobre o ciclo menstrual, } \\
\text { atividades sexuais, gravidez, ingestão de bebidas } \\
\text { alcoólicas, prática de atividades físicas, terapias } \\
\text { complementares, preparo dos alimentos e proteção das } \\
\text { veias. }\end{array}$ & 04 \\
\hline Anotações & $\begin{array}{l}\text { Espaço destinado às anotações, lembretes, marcação do } \\
\text { dia de administração do quimioterápico. }\end{array}$ & 01 \\
\hline Referências & Lista da literatura consultada & 01 \\
\hline
\end{tabular}

Fonte: Dados da pesquisa.

\section{Conclusões}

O câncer vem sendo caracterizado como um grave problema crônico de saúde pública, com caráter cada vez mais estigmatizante e associado frequentemente com os mais diversos fatores deletérios ao ser humano.

Diante do cenário atual em que o atendimento oncológico ambulatorial está cada vez mais difundido, este estudo mostrou a necessidade de somar esforços para garantir aos pacientes as informações necessárias ao enfrentamento de sua patologia, respeitando, principalmente, sua capacidade de compreensão, objetivando torná-los menos ansiosos e apreensivos sobre seu tratamento e incentivando o autocuidado para a garantia da continuidade da assistência em casa.

É neste ponto que é evidenciada a relevância dos materiais educativos, pois eles favorecem o processo interativo entre enfermeiro, paciente ou familiar, auxiliam no diálogo e proporcionam a valorização da humanização da assistência.

Um ponto positivo verificado com este estudo foi a existência de vários materiais acerca do tema em estudo, sejam eles pesquisas de campo ou manuais e protocolos disponibilizados por diversas instituições de saúde que lidam com o tratamento do câncer. Cabe aos profissionais de saúde, em especial ao enfermeiro, selecionar os conteúdos necessários e úteis aos pacientes oncológicos e socializa-los da maneira mais clara possível, sendo este o foco deste trabalho.

Tendo em vista os resultados obtidos por este trabalho é possível o desenvolvimento de sugestões para os gestores da Unacon Dr. Vitor Moutinho, em Tucuruí-PA, a fim de contribuir para o processo de estruturação do serviço de enfermagem na unidade, por meio das ações: após a aprovação da cartilha elaborada realizar o processo de validação da mesma, por meio da avaliação de juízes e público-alvo (pacientes e 


\section{ijhe}

profissionais envolvidos); elaborar programa de treinamento especializado para equipe de enfermagem, pois, é necessário que o enfermeiro esteja capacitado para abordar todos os assuntos pertinentes junto ao paciente e atualizar frequentemente a cartilha educativa; e criar comissões para a elaboração de outros manuais, normas, rotinas e protocolos assistenciais relacionados à quimioterapia, a fim de padronizar as atividades realizadas e orientar a população quanto ao serviço oferecido na unidade oncológica.

\section{Referências}

1. Instituto Nacional de Câncer José Alencar Gomes da Silva (INCA). Estimativa 2014: incidência de câncer no Brasil. Rio de Janeiro: INCA; 2014.

2. Smeltzer SC, Bare BG. Brunner \& Suddarth tratado de enfermagem médico-cirúrgica. 11. ed. Rio de Janeiro: Guanabara Koogan; 2009. (vol. 1).

3. Soffiatti NRT. Consulta de enfermagem em ambulatório de quimioterapia: ênfase nas ações educativas. Cogitare Enferm. 2000;5:69-72 [citado em 2017 Ago 15]. Disponível em: http://revistas.ufpr.br/cogitare/article/view/44872/27296

4. Sousa GR. Tecnologia educativa em saúde para pacientes em tratamento hemodialítico [monografia]. Picos: Universidade Federal do Piauí; 2012 [citado em 2014 Fev 20]. Disponível em: http://www.ufpi.br/subsiteFiles/picos/ arquivos/files/ TCC\%20NOVO.pdf

5. Prado ML, Backes VMS, Reibnitz KS, et al. Produções tecnológicas em enfermagem em um curso de mestrado. Texto Contexto Enferm. 2009; 18(3):475-81. http://dx.doi.org/10.1590/S0104-07072009000300010.

6. Instituto Nacional de Câncer José Alencar Gomes da Silva (INCA). Ações de enfermagem para o controle do câncer: uma proposta de integração ensino-serviço. 3. ed. Rio de Janeiro: INCA; 2008.

7. Camelo SHH, Pinheiro A, Campos D, Oliveira TL. Auditoria de enfermagem e a qualidade da assistência à saúde: uma revisão de literatura. Rev. Eletr. Enf. 2009;11(4):1018-25. http://dx.doi.org/10.5216/ree.v11i4.33258.

8. Salles PC, Castro RCBR. Validação de material informativo a pacientes em tratamento quimioterápico e aos seus familiares. Rev Esc Enferm USP. 2010;44(1):182-9. http://dx.doi.org/10.1590/S0080-62342010000100026.

9. Bonassa EMA, Gato MIR. Terapêutica oncológica para enfermeiros e farmacêuticos. 4. ed. São Paulo: Atheneu; 2012.

10. Barbosa LG, Telles PCP Fo. Conhecimento de pacientes oncológicos sobre a quimioterapia. Cienc Cuid Saúde. 2008;7(3):3705 [citado em 2014 Abr 29]. Disponível em: http://periodicos.uem.br/ojs/index.php/CiencCuidSaude/article/download/ $6510 / 3864$

11. Costa RBC, Leão SS, editores. Manual prático para consulta de enfermagem em quimioterapia antineoplásica. Maceió: Edufal; 2011.

\section{Contribuição dos autores}

Os autores participaram do desenvolvimento do estudo e realizaram a redação e a revisão crítica do mesmo. 